\title{
Interleukin 18 , soluble cluster of differentiation 40 , platelet factor 4 variant 1, and neutrophil gelatinase- associated lipocalin can be used as biomarkers to aid activity and diagnosis in ocular Behçet's disease
}

\section{Fatih Celik}

Elazıg Fethi Sekin City Hospital: Elazig Fethi Sekin Sehir Hastanesi

\section{Ebru Coteli}

Ahi Evran Universitesi

\section{Fatih Cem Gul}

Elazıg Fethi Sekin City Hospital: Elazig Fethi Sekin Sehir Hastanesi

\section{Ercan Ozsoy}

Haseki EAH: Istanbul Haseki Egitim Ve Arastirma Hastanesi

\section{Sabiha Gungor Kobat}

Elazıg Fethi Sekin City Hospital: Elazig Fethi Sekin Sehir Hastanesi

\section{Ramazan Fazil Akkoc}

Fırat Üniversitesi Tıp Fakültesi: Firat Universitesi Tip Fakultesi

\section{Meltem Yardim}

Yozgat Yerkoy State Hospital: Yozgat Devlet Hastanesi

İbrahim Sahin

Erzincan Binali Yildirim University: Erzincan Binali Yildirim Universitesi

\section{Mehmet Hanifi Yalcin}

Fırat Üniversitesi: Firat Universitesi

SÜLEYMAN AYDIN ( $\nabla$ saydin1@hotmail.com )

Firat University School of Medicine: Firat Universitesi Tip Fakultesi

\section{Research Article}

Keywords: Interleukin, neutrophil gelatinase-associated lipocalin, ocular Behçet's disease, platelet factor, soluble cluster of differentiation

Posted Date: August 20th, 2021

DOl: https://doi.org/10.21203/rs.3.rs-812534/v1 
License: (c) (i) This work is licensed under a Creative Commons Attribution 4.0 International License. Read Full License

Version of Record: A version of this preprint was published at International Ophthalmology on May 27th, 2022. See the published version at https://doi.org/10.1007/s10792-022-02331-4. 


\section{Abstract \\ Purpose}

The molecules human interleukin (IL-18), the soluble cluster of differentiation (sCD40), platelet factor 4 variant 1 (PF4V1), and neutrophil gelatinase-associated lipocalin (NGAL) are all markers of inflammation in biological systems and are linked to prognosis in several inflammatory diseases as well. Since there is no study in which the above-mentioned molecules are studied together in ocular Behçet's disease (OBD), the aim of this study is to reveal whether these molecules are activity markers in active (OABD) and inactive (OIBD) disease.

\section{Methods}

$30 \mathrm{OABD}$ and 30 OIBD and 30 healthy individuals were included in the study. IL-18, sCD40, PF4V1, and NGAL molecules were studied in blood samples by the ELISA method.

\section{Results}

When OABD and OIBD were compared to healthy individuals, the levels of IL-18, sCD40, PF4V1, and NGAL molecules were found to be statistically significant. These values were even more significantly higher in patients with OABD.

\section{Conclusion}

When ROC values of IL-18, sCD40, PF4V1, and NGAL are evaluated, it is clear that these four molecules can be used as biomarkers to aid activity and diagnosis in OBD.

\section{Introduction}

Behçet's disease (BD) was first identified in 1937 by the Turkish Dermatologist Hulusi Behçet (1889$1948)[1,2]$. BD is a vascular-inflammatory multisystemic disease characterized by recurrent attacks of oral and genital aphthae, cutaneous lesions, arthritis, gastrointestinal system, central nervous system involvement, and also eye lesions. When BD in terms of eyes, it is seen that ocular involvement is a common event from childhood to old age [3]. Ocular involvement associated with BD is a visionthreatening disease that affects $60-80 \%$ of BD patients and is characterized by iridocyclitis with hypopyon, vitritis, retinal vasculitis, and retinitis, causing structural damage to the eye tissue by damaging the retina and optic nerve [4]. The etiology of BD is yet to be thoroughly elucidated. However, in recent years, the tumor, interferon (IFN) -gamma, tissue necrosis factor (TNF) -alpha, interleukin (IL) IL-1, IL- 6, IL-8, IL-17, and IL-18 have been shown to play significant roles and have direct associations with BD in many studies, putting aside factors such as age, gender, infectious, psychological, and genetics, which 
are considered to be involved in the etiology of BD [5-7]. IL-18 has been reported to be a potent proinflammatory immunomodulatory cytokine that modulates both natural and adaptive immune systems, increases cytokine and chemokine production, stimulates neutrophil and lymphocyte chemotaxis, and IFN-production by natural killer (NK) cells [8, 9]. Previous studies have shown that IL-18 levels are higher in $\mathrm{BD}$ patients compared to the control group, or there is a positive correlation between serum IL-18 levels and $B D$ disease activity and/or site of uptake $[10,11]$. Both macrophages and T cells increase CD40 cytokine output in inflammatory events $[12,13]$. Platelets are activated as a result of these events, and there is a direct link between activated platelets and the inflammatory reaction [14]. Therefore, cytokines secreted by T helper- 1 and 2 cells of the immune system interact with each other in BD [15]. Platelet factor 4 variant 1 (PF4V1) is another cytokine abundant in alpha granules of platelets belonging to the CXC family of chemokines, also known as chemokine ligand 4. PF4V1 inhibits the anticoagulant activity of heparin, is chemotic for neutrophils, monocytes, and fibroblasts, and also inhibits angiogenesis [16]. This cytokine, like other cytokines, is produced de novo in a short period of time in response to immune stimulation in inflammatory conditions, however they are not stored [17]. PF4V1 has been implicated in a number of inflammatory disorders, including liver fibrosis, as a pleiotropic inflammatory chemokine [18]. Studies conducted to date have shown that patients with ocular active Behçet's disease (OABD) and ocular inactive Behçet's disease (OIBD) have increased levels of IL-18, CD40, and PF4V1 [10, 19, 20].

However, no studies have been conducted to demonstrate the course of these molecules in patients with $\mathrm{OABD}$ and OIBD; therefore it remains a significant research subject in this area.

Another molecule associated with inflammation is neutrophil gelatinase-B linked lipocalin (Lcn2/NGAL) [21]. It was first detected in human neutrophil granules [22]. It has been reported that NGAL synthesis increases from epithelial tissues during inflammation and that the lipocalin superfamily is a $21 \mathrm{kD}$ protein with antimicrobial siderophore binding properties [23].

The increased amount of NGAL in aqueous humor fluids obtained from patients with idiopathic acute anterior uveitis was linked to the regulation of inflammation in a previous study, and it was proposed that it could be used as an oculus biomarker. [24]. In addition, this protein has also been reported to be a useful biomarker for early detection of cancers [25], inflammatory bowel disease [26], nephritis [27], acute kidney injury (AKI) [28], heart failure [29], autoimmune myocarditis [30], polyps [31], preeclampsia [32], arthritis [33], and pancreatitis [34]. When recalled that OABD and OIBD is a vascular-inflammatory disease, it is possible that it may have a connection with NGAL. There is a thesis study on OIBD, which is a drug on this subject. Since the amount of NGAL in OABD and OIBD has not been investigated yet, this parameter was also included in this study.

When we combine all information, we can conclude that; although the erythrocyte sedimentation rate (ESR), C-reactive protein (CRP), antistreptolysin O (ASO), alpha-1 antitrypsin, and alpha-2 macroglobulin neutrophil/lymphocyte ratio (NLR) have been identified as diagnostic parameters in the diagnosis of BD $[7,35,36]$, there are currently no clear laboratory test parameters to diagnose the disease. Various scoring systems have recently been established to evaluate the activity and seriousness of BD in patients. 
Tanaka et al. reported a new scoring system for BD-associated uveitis called Behçet's disease ocular attack score 24 (BOS24) [37]. However, as the diagnosis is based on the clinic at present, specific laboratory tests are needed to make the diagnosis fast and based on evidence. Therefore, this study aims to measure the amounts of IL-18, CD40, PF4V1, and NGAL used as inflammation markers and reveal whether they can be easy, cheap, and fast activity markers of OABD and OIBD.

\section{Materials And Methods}

The study was conducted in accordance with the principles of the Declaration of Helsinki by obtaining the approval of the Fırat University Faculty of Medicine, Non-invasive Local Ethics Committee (dated 01.08.2019, meeting number 12 and decision number 15) and informed consent from the participants. The research included 30 patients with OABD and 30 patients with OIBD who were followed up in the Ophthalmology Department, as well as 30 healthy control subjects who were age and gender-matched. The diagnosis of ocular BD was based on the criteria determined by the International Study Group (ISGC) in 1990, and patients with a score of 4 or more were diagnosed with BD [38]. The distinction between active and remission periods was evaluated using Behçet's Disease Current Activity Form (BDCAF), taking into account the clinical criteria defined by Hamuryutan et al. BDCAF was applied to all BD patients participated in the study [39]. These patients were then classified into two categories based on their activity criteria: active and remission periods. Complete blood count, ESR, and CRP values of the patients were scanned retrospectively. Participants with a diagnosis of acute and chronic renal failure, presence of acute and chronic inflammatory disease, and white blood cell count between 4000 and $1200 \mathrm{~h} / \mu \mathrm{l}$, or using antihypertensive, antihyperlipidemic drugs, aspirin, oral contraceptives, systemic steroids or immunosuppressive agents, vitamins, and hormones in the last 4 weeks, alcohol and cigarettes (All participants were nonsmokers and nondrinkers) or with a diagnosis of Diabetes Mellitus, a diagnosis of hypertension, a diagnosis of heart failure, a diagnosis of liver failure, and a history of coronary artery disease, vitamin B12 or folic acid deficiency, history of trauma and body mass index over $30 \mathrm{~kg} / \mathrm{m}^{2}$ in the last two weeks were not included in the study. Another word, BD is a multisystemic inflammatory disease of oral and genital aphthae, cutaneous lesion, arthritis, gastrointestinal system, central nervous system involvement, and ocular lesion. This study is only included ocular lesion patients and control subjects. Following an overnight fast, blood was drawn from all participants into $5 \mathrm{ml}$ plain biochemistry tubes. Controls and BD samples were collected during the same time period. These blood samples were centrifuged for 5 minutes at 4000 RPM. Until they were analyzed, the samples were kept at $-80^{\circ} \mathrm{C}$. All participants had detailed ophthalmological examinations, including best-corrected visual acuity (BCVA), slit-lamp biomicroscopy, and binocular indirect ophthalmoscopy as well. Fundus fluorescein angiography (FFA) (Canon CX-1, Tokyo, Japan) and spectral-domain optical coherence tomography (SD-OCT) (Canon HS100, Tokyo, Japan) images were performed when indicated. Six ocular inflammation symptom parameters were included in BOS24, including anterior chamber cells (maximum 4 points), vitreous opacity (maximum 4 points), peripheral fundus lesions (maximum 8 points), posterior pole lesions (maximum 4 points), foveal lesions (maximum 2 points), and optic disc lesions (maximum 2 points) [37]. 


\section{Analysis of the Studied Molecules by ELISA Method}

Human Interleukin (IL-18) (Catalog no: 201-12-0148), Neutrophil gelatinase-associated lipocalin (NGAL) (Catalog \# 201-12-1720), Soluble Cluster of differentiation (sCD40) (Catalog no: SRB-T-88907), Platelet Factor 4 Variant 1 (PF4V1) (Catalog no: 201-12-4574), kits in blood samples of Ocular BD group and control group were obtained from Sunred Bioscience (Shanghai, CHINA). ELISA measurements were carried out in line with the procedures specified in the catalogs of the kits. All the ELISA tests were at the same time point. For plate washing, the Bio-Tek ELX50 automatic washer (BioTek Instruments, USA) was used, and for absorbance readings, a ChroMate, Microplate Reader P4300 device (Awareness Technology Instruments, USA) was used. The measurement range (assay range) of the IL-18 ELISA kit was $0.6 \mathrm{ng} / \mathrm{L}-$ $100 \mathrm{ng} / \mathrm{L}$, minimum measurable level (sensitivity) was $0.537 \mathrm{ng} / \mathrm{L}$, while the assay range of the NGAL ELISA kit was $12 \mathrm{ng} / \mathrm{mL}-3000 \mathrm{ng} / \mathrm{mL}$, the minimum measurable level (sensitivity) was $10.511 \mathrm{ng} / \mathrm{mL}$. The assay range of the $S C D 40$ ELISA kit was $0.1 \mathrm{ng} / \mathrm{mL}-30 \mathrm{ng} / \mathrm{mL}$, the minimum measurable level (sensitivity) was $0.076 \mathrm{ng} / \mathrm{mL}$, while the assay range of the PF4V1 ELISA kit was $25 \mathrm{ng} / \mathrm{L}-7000 \mathrm{ng} / \mathrm{L}$, the minimum measurable level (sensitivity) was $24.246 \mathrm{ng} / \mathrm{L}$. Additionally, all the kits used here had an IntraAssay CV value of $<10 \%$ and an Inter-Assay CV value of $12 \%$.

\section{Statistical Analyses}

Statistical analyzes were performed using the "Statistical Packages for the Social Sciences" (SPSS) version 24.0 package program (SPSS Inc., Chicago, IL, USA). Whether there is a difference between the distribution of descriptive parameters such as age, body mass, and index between the patient and control groups was analyzed using the chi-square test, and whether there was a difference between the values of the CRP and hemogram parameters measurements of the patient and control group participants were analyzed with the Kruskal Wallis test, and Kolmogorov-Smirnov test was used to check the normality of the sample distribution. ANOVA test was used when comparing patient and control groups with patient subgroups and laboratory parameters. Duncan's new multiple range test was applied between the groups with statistical differences and $p<0.05$ was considered statistically significant. Receiver operating characteristic (ROC) analysis was used to measure diagnostic performance parameters, and areas under the curve were determined as $95 \%$ confidence intervals.

\section{Results}

The demographic and some biochemical findings of the control and patient groups are provided in Table 1. When the groups were evaluated in terms of neutrophil levels, it was observed that it was $3.82 \pm$ 1.72 in the control group, $5.62 \pm 1.84$ in the OIBD group, and 7.22 \pm 1.84 in the OABD group. Neutrophil levels were found to be statistically significantly higher in the OABD group compared to the OIBD group and the healthy control group $(p<0.002)$ (Table 1$)$. Once again, in the OABD group, the CRP levels were found to be statistically significantly higher than the OIBD group and the healthy control group $(p<0.001)$ (Tablo 1). There was a significant difference in BOS24 and BDCAF scores between the groups and the scores were as follows: $13.06 \pm 2.35$ and $3.75 \pm 2.01$ in OABD group and $0.25 \pm 44$ and $1.88 \pm 2.33$ in OIBD 
group, respectively $(p<0.001)$. While the BOS24 score was $14.01 \pm 2.23$ and the BDCAF value was $3.63 \pm$ 1.94 in the OABD group, the BOS24 score was $0.28 \pm 023$ and the BDCAF value was $1.94 \pm 2.2$ in the OIBD group. These BOS24 and BDCAF scores were statistically significantly different between the groups. IL-18 (Fig. 1), sCD40 (Fig. 2), PF4V1 (Fig. 3), and NGAL (Fig. 4) were higher in OABD group and OIBD group than in the healthy control group $(p<.005)$. Besides, the amounts of IL-18 (Fig. 1), sCD40 (Fig. 2), PF4V1 (Fig. 3), and NGAL (Fig. 4) were higher in OABD group, and when OABD group and OIBD group were compared with each other in terms of these parameters, there was a statistically significant difference. Moreover, in patients with OABD group, there was a positive correlation between the amount of IL-18, sCD40, PF4V1, and NGAL and the amount of CRP. Also, there was a positive correlation between the amounts of IL-18, sCD40, PF4V1, and NGAL and the BOS24 score in patients with OABD group. When evaluating the diagnostic performance of OABD group with the ROC curve, it was determined as IL-18 (specificity: and sensitivity:), sCD40 (specificity: and sensitivity:), PF4V1 (specificity: and sensitivity:) and NGAL (specificity: and sensitivity:). The evaluations regarding the diagnostic performance of these parameters are presented in detail in Table 2 and Fig. 5.

Table 1

Comparison of demographic and some biochemical parameters of the subjects.

\begin{tabular}{|lllll|}
\hline & Control & OIBD & OABD & $p$ value \\
\hline Sex $(\mathrm{M} / \mathrm{F})$ & $15 / 15$ & $18 / 12$ & $17 / 13$ & $0.877^{\star}$ \\
\hline Age $($ years $\pm \mathrm{SD})$ & $35.2 \pm 4.9$ & $36.3 \pm 5.6$ & $35.9 \pm 4.2$ & $0.963^{\star \star}$ \\
\hline WBC count $\left(\mathrm{K} / \mathrm{mm}^{3}\right)$ & $7.12 \pm 2.2$ & $7.63 \pm 2.9$ & $8.02 \pm 4.1$ & $0.462^{\star \star}$ \\
\hline Neutrophil $\left(10^{3} / \mu \mathrm{l}\right)$ & $3.82 \pm 1.72$ & $5.62 \pm 1.84$ & $7.22 \pm 1.84$ & $<0.002^{\star \star}$ \\
CRP $(\mathrm{mg} / \mathrm{L})$ & $1.7 \pm 0.11$ & $2.2 \pm 0.22$ & $4.9 \pm 0.61$ & $<0.001^{\star *}$ \\
\hline OIBD: Ocular inactive Behcet disease; OABD: Ocular active Behcet disease; WBC: white blood cell. \\
\hline *Chi-square test; **One-way analysis of variance test. & & \\
\hline
\end{tabular}

Table 2

Comparison of diagnostic performances of variables with each other.

\begin{tabular}{|llllll|}
\hline Risk Factor & AUC 95\% (Cl) & Cut off & $p$ value & Sensitivity (\%) & Specificity (\%) \\
\hline IL-18 & $0.998(0.994-1.000)$ & 13.83 & 0.002 & 95.8 & 97.9 \\
\hline NGAL & $1.000(1.000-1.000)$ & 355.55 & 0.000 & 100.0 & 97.9 \\
\hline PF4V1 & $0.988(0.964-1.000)$ & 624.66 & 0.012 & 100.0 & 97.9 \\
\hline SCD40 & $0.986(0.965-1.000)$ & 2.36 & 0.000 & 70.8 & 97.9 \\
\hline $\begin{array}{l}\text { AUC: Area under the curve; Cl: Confidence Interval; IL-18: Interleukin-18; NGAL: Neutrophil gelatinase- } \\
\text { associated lipocalin; PF4V1: Platelet factor-4 Variant 1; sCD40: soluble cluster of differentiation 40. }\end{array}$ \\
\hline
\end{tabular}




\section{Discussion}

There is currently no exact specific marker to determine BD's inflammatory activity. Hence, it was revealed whether IL-18, sCD40, PF4V1, and NGAL inflammation biomarkers in the blood of individuals with OABD and OIBD would be useful in the diagnosis of the disease. Cytokines play a key role in the pathophysiology of BD [40]. In this current study, it was discovered that IL-18, a cytokine, increased in ocular active and inactive Behçet patients in a statistically significant way as compared to the control group values. The increase in IL-18 was more pronounced in patients with OABD than in the other two groups. The source of the IL-18 increases reported here may be monocyte and macrophage cells activated due to BD. This is because inflammation is one of the main symptoms in BD, and in this case, it has been reported that monocytes and macrophages produce a series of pro-inflammatory cytokines [6, $40,41]$. It has been previously reported that the amount of circulating IL-18 increases during BD regardless of ocular manifestation [10]. Considering a previously reported report with available data [10], it can be suggested that increases in IL-18 may be indicative of inflammatory ocular lesions. The $95 \%$ confidence interval under the curve in the ROC curve analysis recorded for the first time in our study, as well as the associated $p$-value, suggest that IL-18 may be a useful parameter in diagnosing active and inactive BD. Moreover, in previous studies, some cytokine releases of active and inactive Behçet patients and healthy individuals were compared, and it was reported that some cytokines such as IL-6, IL-12, and IFN- $\gamma$ were significantly increased in active and inactive Behçet patients according to the values of healthy individuals [42]. Furthermore, IL-18Ra is produced in almost all immune cells and some nonimmune cells [43]. Therefore, IL-18 has the potential to be an important laboratory parameter in the future in determining the inflammation processes of ocular BD, as it has roles in immune complex-mediated pathology, potentially affecting both congenital and adaptive immune responses [6].

In this study, SCD40 values in the blood of individuals with active and inactive ocular BD were studied and compared with the control group. A significant increase was found in CD40 ocular active and inactive Behçet patients compared to control values. The highest increase was recorded in individuals with OABD. CD40 plays a role in other immune and vascular pathologies, including BD. In particular, previously published data reported that platelet-derived CD40 levels were higher in Behçet's patients than in healthy control groups [19]. These available data, which are identified for the first time in OABD and OIBD, indicate that ocular inflammation may be a significant biomarker in evaluating disease activity, according to our comprehensive available study. This is because CD40 is an innate transmembrane molecule that plays an important role in adaptive immunity [44]. Hence, the increase in CD40 in ocular BD may also be an ocular reflection of systemic disease. In other words, it is predicted that CD40, which is increased in ocular BD, may have roles in the physiopathology of ocular BD. The $95 \%$ confidence interval under the curve in the ROC curve analysis recorded in our study, as well as the related $p$-value, suggest that CD40 may be a useful additional parameter for diagnosing OABD and OIBD.

In this study, it was found that PF4V1 amounts in OABD and OIBD were significantly increased when compared with the values of the control group, and this increase was even higher in OABD. Increased amounts of the PF4V1 molecule in OABD may be an indication that the platelets (thrombocytes) interact 
with leukocytes (monocytes, neutrophils, dendritic cells, T-cells) and progenitor cells to encourage the migration of inflammatory cells to the lesion areas, resulting in the release of a large number of inflammatory cytokines and ultimately an inflammatory environment in the lesion area [45]. Thus, the increase in IL-18 and SCD40 values noted in the upper section of the discussion in OABD and OIBD may have been due to an increase in leukocyte migration caused by platelets (thrombocytes) activation in cases of inflammation [45]. For instance, activated monocytes and macrophages are the key cellular source of IL-18 [46]. Similarly, it has been reported that leukocyte migration causes thrombocyte activation due to inflammation, which results in the release of inflammatory cytokines (such as IL-6) [47]. Our findings also show that PF4V1 can be a successful biomarker candidate for determining the activity of ocular BD based on its ROC values.

In this study, when the OABD and OIBD and control group values were compared in terms of NGAL amounts, a statistically significant increase was found in the case of ocular BD. Moreover, NGAL values were significantly even higher in OABD. Tissue distribution and expression in tissues exposed to microorganisms such as neutrophils, bone marrow and trachea, lung, stomach, salivary gland, and colon [48] indicate that NGAL plays significant roles in inflammatory responses. In neutrophils, NGAL secretion is regulated by activation of infection and inflammation [49]. The possible reason for the further increase of NGAL amounts in OABD and OIBD in our study may be due to the sequestration of neutrophil chemoreactants due to inflammation in the eye, and the amount may be increased to reduce the neutrophil-dependent inflammatory response. It has been previously reported that NGAL amounts are increased in other inflammatory diseases such as Rheumatoid Arthritis (RA) [33] and inflammatory bowel disease [26]. On the other hand, in a thesis study conducted in 2012, it was recorded that the amount of NGAL in BD decreased, ${ }^{50}$ and these results are inconsistent with the NGAL results of our ocular BD study, which we recorded here. The same researcher also reported that there was no statistically significant difference between the serum NGAL values of Behçet's patients with ocular involvement and healthy individuals [50]. However, they did not include the OABD group in their study. Besides, this researcher stated that Behçet's patients used one or more agents of colchicine, systemic steroids, or immunosuppressive [50]. However, the use of any medication in our patients was the exclusion criterion from the study. In addition, there is no other study evaluating NGAL results in Behçet's eye disease in the literature. Therefore, the contrast between the aforementioned study and our study in terms of NGAL values may be due to drug use [50]. Our ROC curve analysis results also indicate that NGAL values in $\mathrm{OABD}$ and OIBD may be associated with the etiology of this disease and may be an inflammatory biomarker.

Moreover, in this study, only the systemic activity score was evaluated in ocular active and inactive patients using BDCAF. BDCAF scores of the patients included in the study were lower. Our BDCAF score results reported here are consistent with the results of Balbaba et al., where Serum Cortistatin levels were reported in patients with OABD and OIBD. ${ }^{7}$ As Balbaba et al. suggested in their studies [7], we predict that it may be more valuable to evaluate IL-18, SCD40, PF4V1, and NGAL amounts, particularly in periods when systemic findings indicate a higher BDCAF score. 
This study has also some limitations as every study has. The main limitation of this study is its crosssectional design and relatively small sample size. In several previous studies, it was tried to be associated with the disease by examining a single biological molecule. Looking at a molecule is no longer appropriate for disease diagnosis and treatment in the proteomic era we live in. Although 4 diseaserelated molecules were studied in this study, we think that the number of molecules was still insufficient, and we accept that this is another limitation of our study. In the future, studying a large number of molecules associated with diseases will save journals pages and time. Furthermore, our broad exclusion criteria prevented us from enrolling more patients in this study.

In conclusion, when all of the evidence from this study were combined, these molecules have increased in the peripheral blood of patients with OABD and OIBD, and they are low-cost, easily measurable new biomarkers that are possibly related to the disease's pathogenesis and can help diagnosis. At the same time, higher detection of these parameters in OABD can be a guide in distinguishing between OABD and OIBD and may contribute to the monitoring of OABD. According to our ROC curve results, the superiority of IL-18, sCD40, PF4V1, and NGAL biomarkers to each other in diagnosing OABD and OIBD was not significant. However, it is also clear that broader and more comprehensive studies are needed to more accurately demonstrate the functions of these IL-18, sCD40, PF4V1, and NGAL biomarkers.

\section{Declarations}

Author contributions FC,SA contributed to concept, design, intellectual content and drafted the article. is contributed to design, concept, and statistical analysis. FC, FCG, SGK, EO acquired data and interpreted data. The whole authors contributed to data analysis and intellectual content. FC acquired data, drafted the article and interpreted data. The whole authors contributed to data analysis.

Funding No financial support was received for this study.

\section{Compliance with ethical standards}

Conflict of interest The authors declare that they have no conflict of interest.

Ethical approval All procedures performed in studies involving human participants were in accordance with the ethical standards of Firat University Faculty of Medicine ethics committee and with the 1964 Helsinki declaration and its later amendments or comparable ethical standards.

Informed consent Informed consent was obtained from all individual participants included in the study.

\section{References}

1. Behçet $H$ (1937) Über rezidivierende, aphtöse, durch ein Virus verursachte Geschwüre am Mund, am Auge und an den Genitalien. Dermatol Wochenschr 105:1152-1163 
2. Turgut YB, Turgut M (2020) Turkish scientist Hulusi Behçet (1889-1948) and his contribution to the medical world. Childs Nerv Syst 36:665-666. https://doi:10.1007/s00381-019-04081-8

3. Tan SY, Poole PS (2016) Hulusi Behçet (1889-1948): passion for dermatology. Singap Med J 57:408-409. https://doi:10.11622/smedj.2016123

4. Bansal R, Gupta V, Gupta A (2010) Current approach in the diagnosis and management of panuveitis. Indian J Ophthalmol 58:45-54. https://doi:10.4103/0301-4738.58471

5. Emmi G, Silvestri E, Squatrito D et al (2014) Emmi L. Behçet's syndrome pathophysiology and potential therapeutic targets. Intern Emerg Med 9:257-265. https://doi:10.1007/s11739-013-1036-5

6. Tong B, Liu X, Xiao J et al (2019) Immunopathogenesis of Behcet's Disease. Front Immunol 10:665. https://doi:10.3389/fimmu.2019.00665

7. Balbaba M, Ulaş F, Postacı SA et al (2020) Serum Cortistatin Levels in Patients with Ocular Active and Ocular Inactive Behçet Disease. Ocul Immunol Inflamm 28:601-605. https://doi:10.1080/09273948.2019.1610461

8. Kany S, Vollrath JT, Relja B (2019) Cytokines in Inflammatory Disease. Int J Mol Sci 20:6008. https://doi:10.3390/ijms20236008

9. Cronkite DA, Strutt TM (2018) The Regulation of Inflammation by Innate and Adaptive Lymphocytes. J Immunol Res 2018:1467538. https://doi:10.1155/2018/1467538

10. Musabak U, Pay S, Erdem H et al (2006) Serum interleukin-18 levels in patients with Behçet's disease. Is its expression associated with disease activity or clinical presentations? Rheumatol Int 26:545550. https://doi:10.1007/s00296-005-0029-8

11. Oztas MO, Onder M, Gurer MA et al (2005) Serum interleukin 18 and tumour necrosis factor-alpha levels are increased in Behcet's disease. Clin Exp Dermatol 30:61-63. https://doi:10.1111/j.13652230.2004.01684.x

12. Kawabe T, Matsushima M, Hashimoto $\mathrm{N}$ et al (2011) CD40/CD40 ligand interactions in immune responses and pulmonary immunity. Nagoya J Med Sci 73:69-78

13. Elgueta R, Benson MJ, de Vries VC et al (2009) Molecular mechanism and function of CD40/CD40L engagement in the immune system. Immunol Rev 229:152-172. https://doi:10.1111/j.1600065X.2009.00782.X

14. Linke B, Schreiber Y, Picard-Willems B et al (2017) Activated Platelets Induce an Anti-Inflammatory Response of Monocytes/Macrophages through Cross-Regulation of PGE2 and Cytokines. Mediators Inflamm 2017:1463216. https://doi:10.1155/2017/1463216

15. Raziuddin S, al-Dalaan A, Bahabri S et al (1998) Divergent cytokine production profile in Behçet's disease. Altered Th1/Th2 cell cytokine pattern. J Rheumatol 25:329-333

16. Fleischer J, Grage-Griebenow E, Kasper B et al (2002) Platelet factor 4 inhibits proliferation and cytokine release of activated human T cells. J Immunol 169:770-777. https://doi:10.4049/jimmunol.169.2.770 
17. Joglekar M, Khandelwal S, Cines DB, ET AL (2015) Heparin enhances uptake of platelet factor 4/heparin complexes by monocytes and macrophages. J Thromb Haemost 13:1416-1427. https://doi:10.1111/jth.13003

18. Kaiko GE, Horvat JC, Beagley KW et al (2008) Immunological decision-making: how does the immune system decide to mount a helper T-cell response? Immunology 123:326-338. https://doi:10.1111/j.1365-2567.2007.02719.x

19. Perazzio SF, Soeiro-Pereira PV, Dos Santos VC et al (2017) Soluble CD40L is associated with increased oxidative burst and neutrophil extracellular trap release in Behçet's disease. Arthritis Res Ther 19:235. https://doi:10.1186/s13075-017-1443-5

20. Schmitz-Huebner U, Knop J (1984) Evidence for an endothelial cell dysfunction in association with Behçet's disease. Thromb Res 34:277-285. https://doi:10.1016/0049-3848(84)90384-0

21. Abella V, Scotece M, Conde J et al (2015) The potential of lipocalin-2/NGAL as biomarker for inflammatory and metabolic diseases. Biomarkers 20:565-571.

https://doi:10.3109/1354750X.2015.1123354

22. Passov A, Petäjä L, Pihlajoki M et al (2019) The origin of plasma neutrophil gelatinase-associated lipocalin in cardiac surgery. BMC Nephrol 20:182. https://doi:10.1186/s12882-019-1380-4

23. Clifton MC, Rupert PB, Hoette TM et al (2019) Parsing the functional specificity of Siderocalin/Lipocalin 2/NGAL for siderophores and related small-molecule ligands. J Struct Biol X 2:100008. https://doi:10.1016/j.yjsbx.2019.100008

24. Salom D, Sanz-Marco E, Mullor JL et al (2010) Aqueous humor neutrophil gelatinase-associated lipocalin levels in patients with idiopathic acute anterior uveitis. Mol Vis 16:1448-1452

25. Li T, Yu L, Wen J et al (2016) An early-screening biomarker of endometrial carcinoma: NGAL is associated with epithelio-mesenchymal transition. Oncotarget 2016;7(52):86064-86074. https://doi:10.18632/oncotarget.13340

26. Thorsvik S, Damås JK, Granlund AV et al (2017) Fecal neutrophil gelatinase-associated lipocalin as a biomarker for inflammatory bowel disease. J Gastroenterol Hepatol 32:128-135. https://doi:10.1111/jgh.13598

27. Suzuki M, Wiers KM, Klein-Gitelman MS et al (2008) Neutrophil gelatinase-associated lipocalin as a biomarker of disease activity in pediatric lupus nephritis. Pediatr Nephrol 23:403-412. https://doi:10.1007/s00467-007-0685-x

28. Shang W, Wang Z (2017) The Update of NGAL in Acute Kidney Injury. Curr Protein Pept Sci 18:12111217. https://doi:10.2174/1389203717666160909125004

29. Damman K, Valente MAE, van Veldhuisen DJ et al (2017) Plasma Neutrophil Gelatinase-Associated Lipocalin and Predicting Clinically Relevant Worsening Renal Function in Acute Heart Failure. Int J Mol Sci 18:1470. https://doi:10.3390/ijms18071470

30. Ding L, Hanawa H, Ota $Y$ et al (2010) Lipocalin-2/neutrophil gelatinase-B associated lipocalin is strongly induced in hearts of rats with autoimmune myocarditis and in human myocarditis. Circ $\mathrm{J}$ 74:523-530. https://doi:10.1253/circj.cj-09-0485 
31. Woo HJ, Min JK, Bai CH et al (2008) Expression of neutrophil gelatinase-associated lipocalin in nasal polyps. Arch Otolaryngol Head Neck Surg 134:1182-1186. https://doi:10.1001/archotol.134.11.1182

32. Artunc-Ulkumen B, Guvenc Y, Goker A et al (2015) Relationship of neutrophil gelatinase-associated lipocalin (NGAL) and procalcitonin levels with the presence and severity of the preeclampsia. $J$ Matern Fetal Neonatal Med 28:1895-1900. https://doi:10.3109/14767058.2014.972926

33. Katano M, Okamoto K, Arito M et al (2009) Implication of granulocyte-macrophage colonystimulating factor induced neutrophil gelatinase-associated lipocalin in pathogenesis of rheumatoid arthritis revealed by proteome analysis. Arthritis Res Ther 11:R3. https://doi:10.1186/ar2587

34. Siddappa PK, Kochhar R, Sarotra P et al (2018) Neutrophil gelatinase-associated lipocalin: An early biomarker for predicting acute kidney injury and severity in patients with acute pancreatitis. JGH Open 3:105-110. https://doi:10.1002/jgh3.12112

35. Uzkeser H, Karatay S, Yildirim K et al (2011) Antistreptolysin O Levels in Patients with Behcet's Disease. Eurasian J Med 43:169-172. https://doi:10.5152/eajm.2011.33

36. Balbaba M, Ulaş F, Yıldırım H et al (2020) Thiol/disulfide homeostasis in patients with ocular-active and ocular-inactive Behçet disease. Int Ophthalmol 40:2643-2650. https://doi:10.1007/s10792-02001445-x

37. Tanaka R, Murata H, Takamoto M et al (2016) Behçet's disease ocular attack score 24 and visual outcome in patients with Behçet's disease. Br J Ophthalmol 100:990-994. https://doi:10.1136/bjophthalmol-2015-307362

38. International Study Group for Behçet's Disease (1990) Criteria for diagnosis of Behçet's disease. Lancet 335:1078-1080. https://doi.org/10.1016/0140-6736(90)92643-V

39. Hamuryudan V, Fresko I, Direskeneli $\mathrm{H}$ et al (1999) Evaluation of the Turkish translation of a disease activity form for Behçet's syndrome. Rheumatology 38:734-736.

https://doi:10.1093/rheumatology/38.8.734

40. Zhou ZY, Chen SL, Shen N et al (2012) Cytokines and Behcet's disease. Autoimmun Rev 11:699-704. doi 10.1016/j.autrev.2011.12.005

41. Park UC, Kim TW, Yu HG (2014) Immunopathogenesis of ocular Behçet's disease. J Immunol Res 2014:653539. https://doi:10.1155/2014/653539

42. Hamzaoui K, Hamzaoui A, Guemira F et al (2002) Cytokine profile in Behçet's disease patients. Relationship with disease activity. Scand J Rheumatol 31:205-210. https://doi:10.1080/030097402320318387

43. Yasuda K, Nakanishi K, Tsutsui H (2019) Interleukin-18 in Health and Disease. Int J Mol Sci 20:649. https://doi:10.3390/ijms20030649

44. Cardone J, Le Friec G, Kemper C (2011) CD46 in innate and adaptive immunity: an update. Clin Exp Immunol 164:301-311. https://doi:10.1111/j.1365-2249.2011.04400.x

45. Kral JB, Schrottmaier WC, Salzmann M et al (2016) Platelet Interaction with Innate Immune Cells. Transfus Med Hemother 43:78-88. https://doi:10.1159/000444807 
46. Arango Duque G, Descoteaux A (2014) Macrophage cytokines: involvement in immunity and infectious diseases. Front Immunol 5:491. https://doi:10.3389/fimmu.2014.00491

47. Tanaka T, Narazaki M, Kishimoto T (2014) IL-6 in inflammation, immunity, and disease. Cold Spring Harb Perspect Biol 6:a016295. https://doi:10.1101/cshperspect.a016295

48. Rosales C (2018) Neutrophil: A Cell with Many Roles in Inflammation or Several Cell Types? Front Physiol 9:113. https://doi:10.3389/fphys.2018.00113

49. Chakraborty S, Kaur S, Guha S et al (2012) The multifaceted roles of neutrophil gelatinase associated lipocalin (NGAL) in inflammation and cancer. Biochim Biophys Acta 1826:129-169. https://doi:10.1016/j.bbcan.2012.03.008

50. Donmez N (2012) Lipocalin 2 levels in patients with Behçet's disease, Kahraman Maras Sutcu İmam University, specialization thesis, Kahramanmaras, Turkey

\section{Figures}

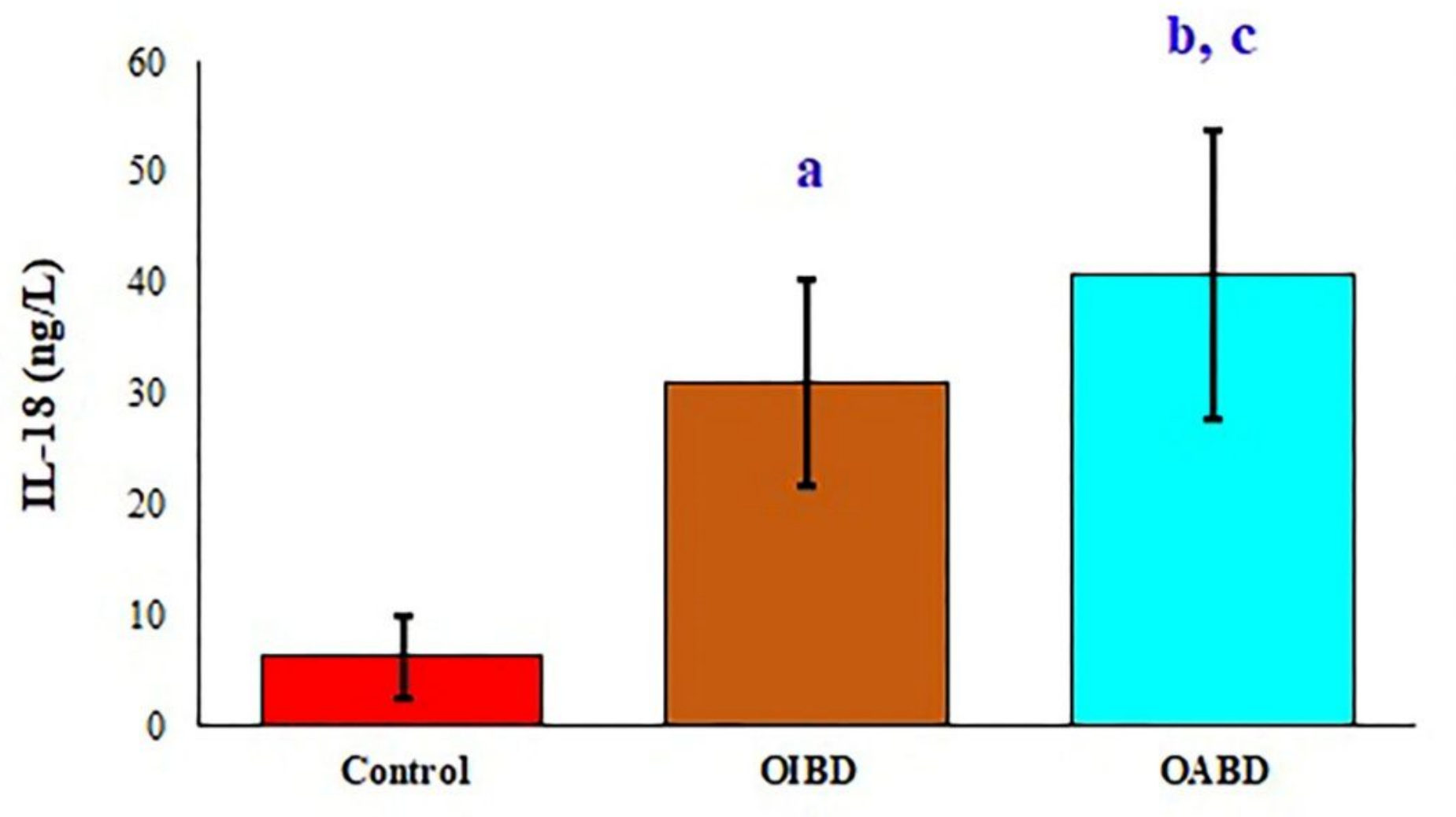

\section{Figure 1}

Comparison of IL-18 amounts with control group values in Inactive and Active Ocular Behçet's Disease. IL-18: Interleukin-18; OABD: ocular active Behçet's disease; OIBD: ocular inactive Behçet's disease. a: Control versus OIBD $(p<0.01)$. b: Control versus OABD $(p<0.01)$. c: OIBD versus OABD $(p<0.01)$. 


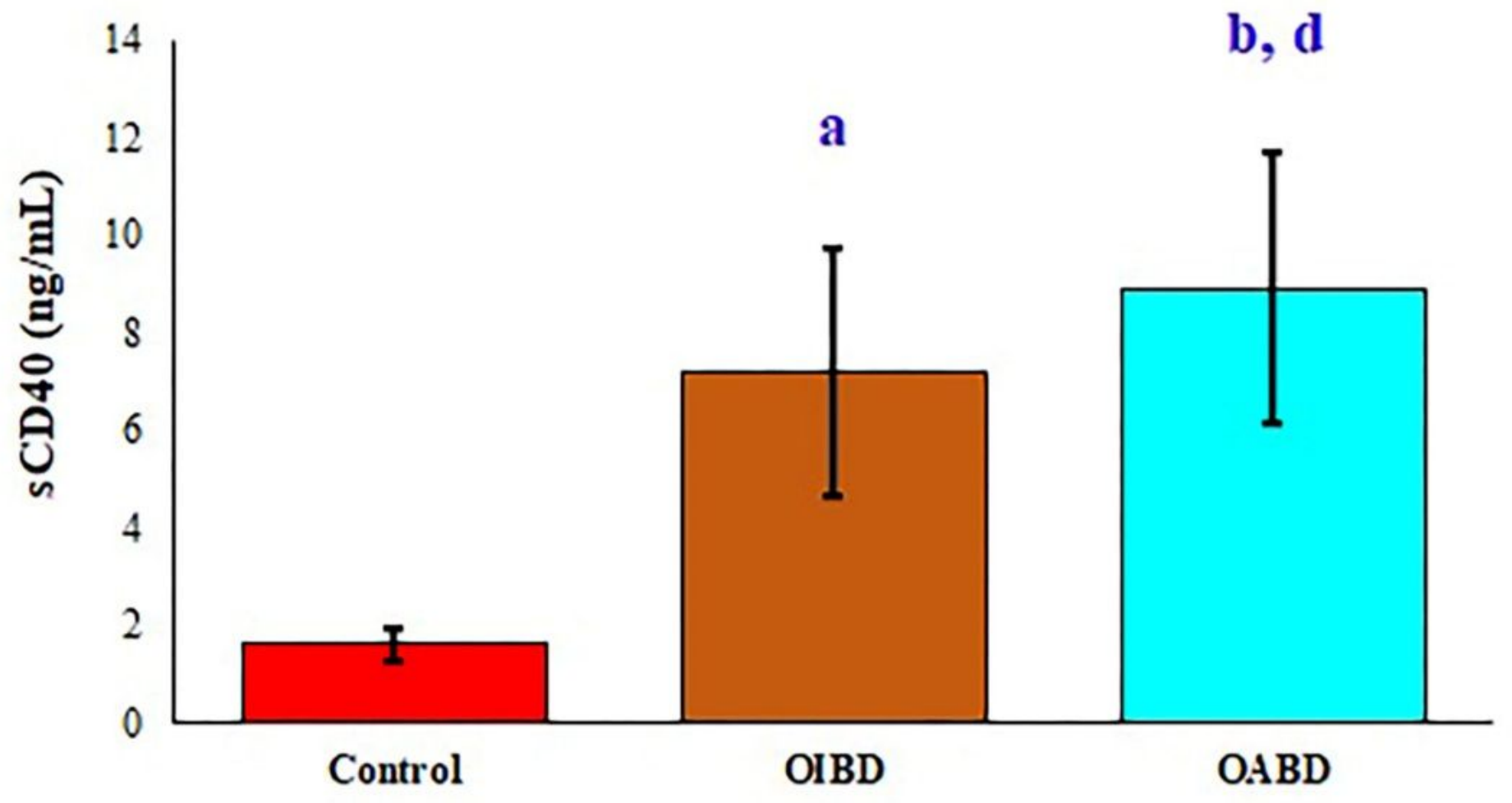

Figure 2

Comparison of SCD40 amounts with control group values in Inactive and Active Ocular Behçet's disease. OABD: ocular active Behçet's disease; OIBD: ocular inactive Behçet's disease; sCD40: soluble cluster of differentiation 40 . a: Control versus OIBD $(p<0.01)$. b: Control versus OABD $(p<0.01)$. d: OIBD versus $\operatorname{OABD}(p<0.05)$. 


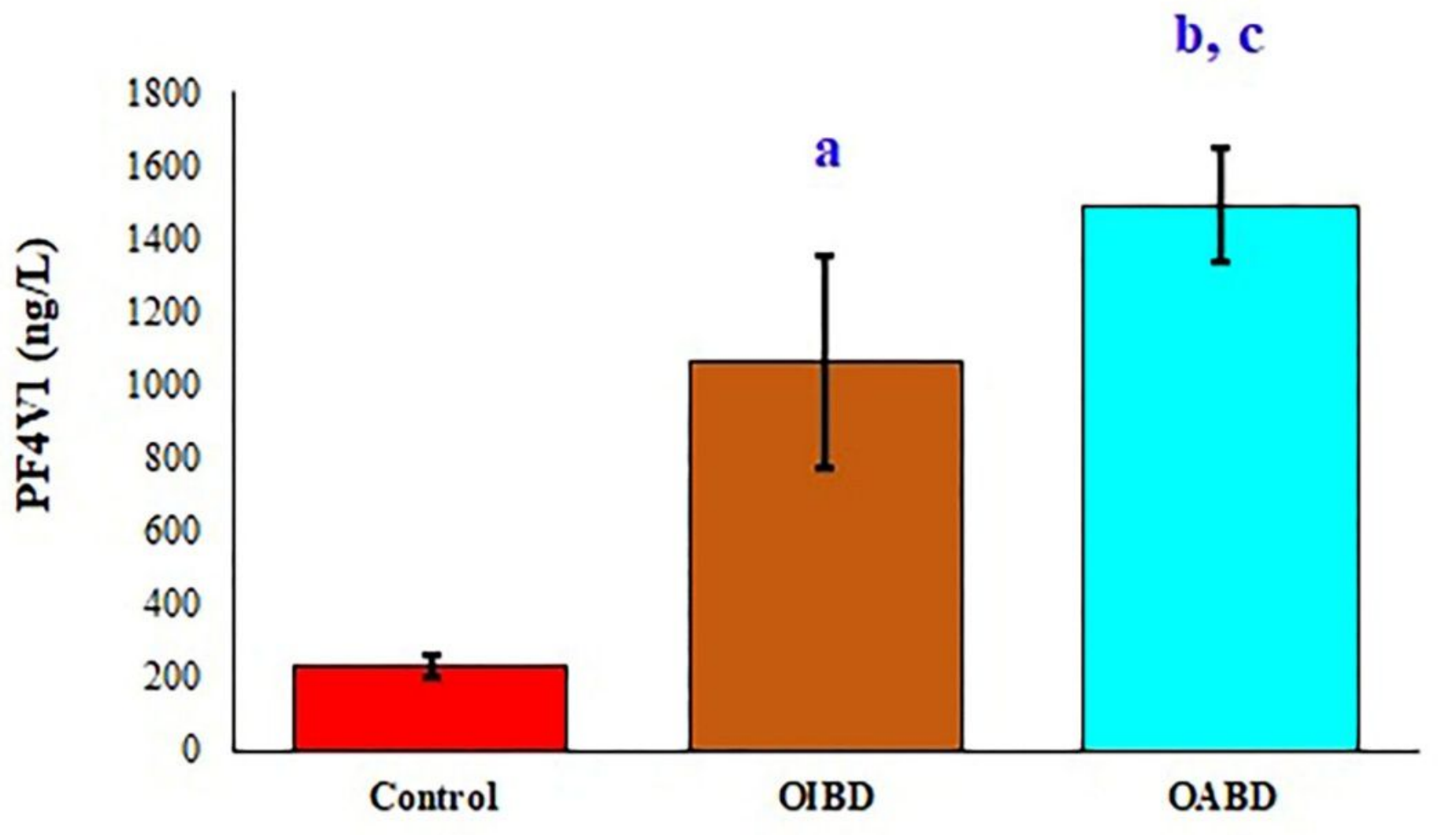

Figure 3

Comparison of PF4V1 amounts with control group values in Inactive and Active Ocular Behçet's disease. OABD: ocular active Behçet's disease; OIBD: ocular inactive Behçet's disease; PF4V1: Platelet factor-4 variant 1. a: Control versus OIBD $(p<0.01)$. b: Control versus OABD $(p<0.01)$. c: OIBD versus OABD $(p<$ $0.01)$. 


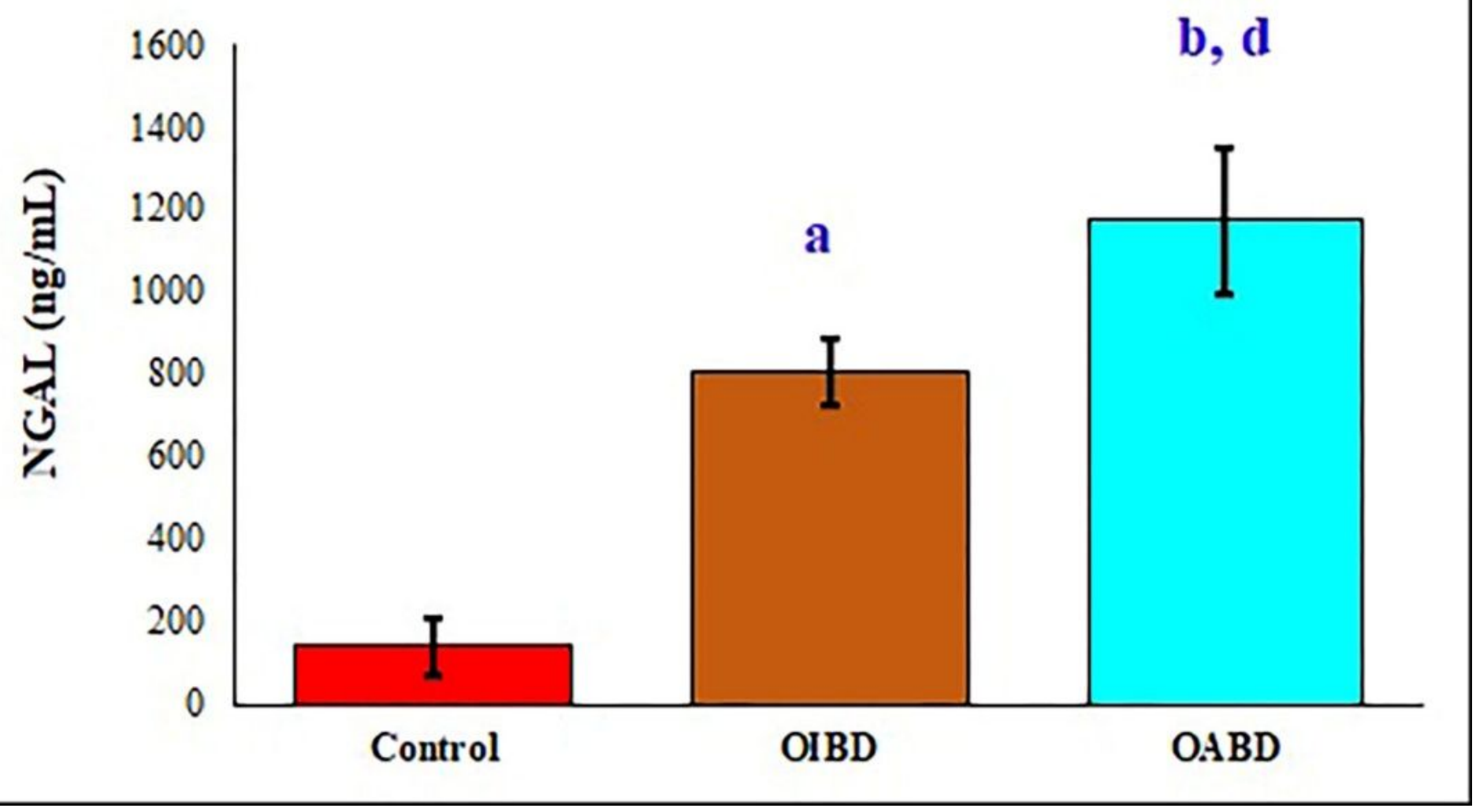

Figure 4

Comparison of Neutrophil gelatinase-associated lipocalin amounts with control group values in Inactive and Active Ocular Behçet's disease. NGAL: Neutrophil gelatinase-associated lipocalin; OABD: ocular active Behçet's disease; OIBD: ocular inactive Behçet's disease. a: Control versus OIBD $(p<0.01)$. $b$ : Control versus OABD $(p<0.01)$. d: OIBD versus OABD $(p<0.05)$. 


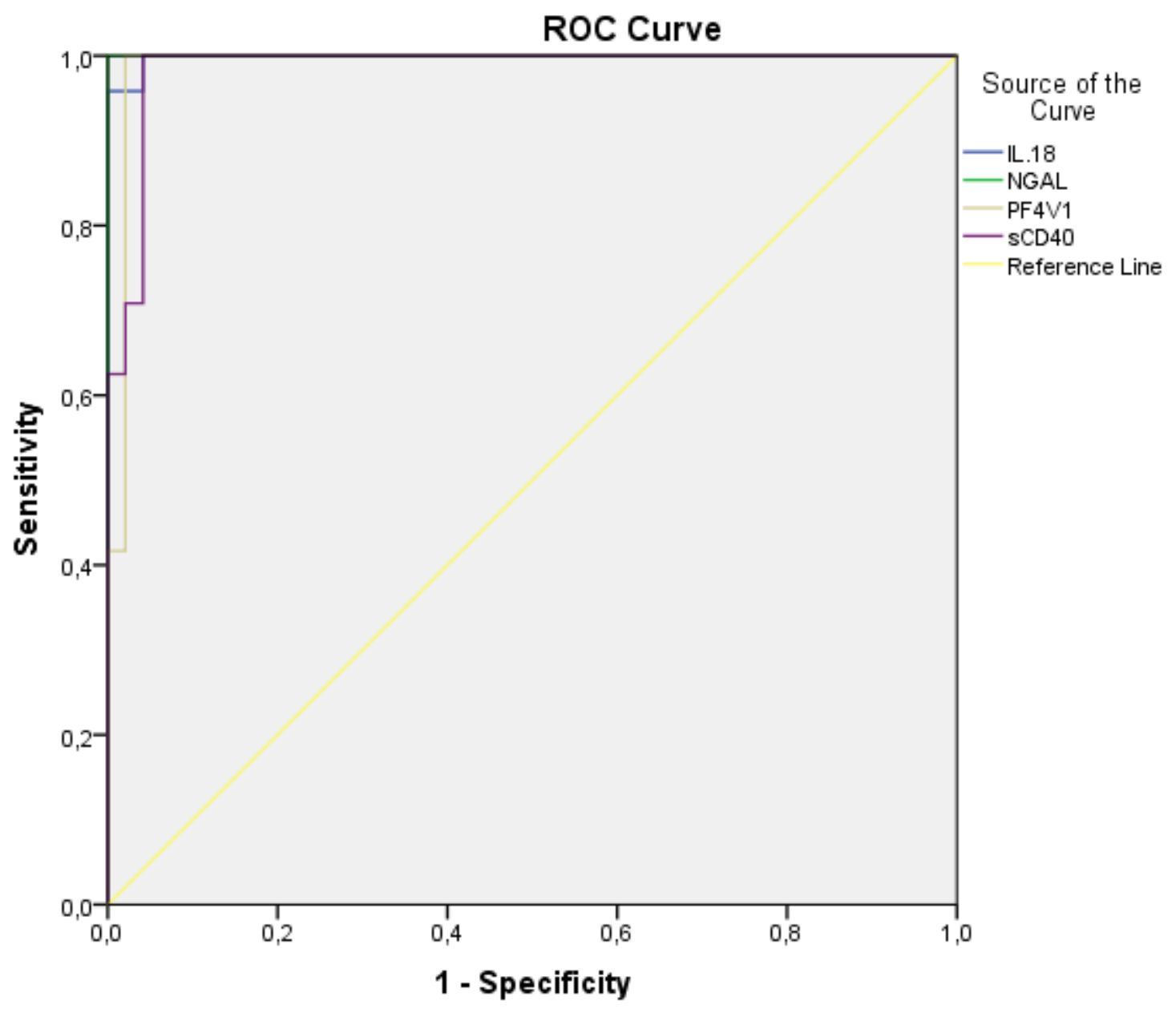

Figure 5

The ability of IL-18, SCD40, PF4V1, and NGAL in the diagnosis of ocular Behçet's disease. IL-18: Interleukin-18; NGAL: Neutrophil gelatinase-associated lipocalin; PF4V1: Platelet factor-4 variant 1; sCD40: soluble cluster of differentiation 40. 